\title{
Altered Biological Properties in Dp71 Over-Expressing HBE Cells
}

\author{
Sipin Tan ${ }^{b}$ Shuai Zhao ${ }^{b, f} \quad Z^{2}$ hikang Chen ${ }^{d}$ Qiongshang Mae Wenmei Wang ${ }^{b}$ \\ Shuhua Cheng ${ }^{b}$ Qiaocheng Wen ${ }^{d}$ Sichuang Tan ${ }^{a} \quad$ Jinliang Xie ${ }^{c}$ \\ aDepartment of Thoracic Surgery, the Second Xiangya Hospital, Central South University, Changsha, \\ Hunan, 'Laboratory of Shock, Department of Pathophysiology, Xiangya School of Medicine, Central \\ South University, Changsha, Hunan, 'Center of Transplant Surgery, Xiangya Hospital, Central South \\ University, Changsha, Hunan, 'Department of General Surgery. Xiangya Hospital, Central South \\ University, Changsha, Hunan, eDepartment of Microbiology, Xiangya School of Medicine, Central South

 \\ Hospital, Tianjin, China
}

\section{Key Words}

Dp71 • HBE • Lamin B1 • FAK /ERK/cyclin D • Biological characters

\begin{abstract}
Background/Aims: In order to further characterize the biological traits of Dp71, HBE over expressing two most abundantly expressed Dp71 spliced isoforms, Dp71d and Dp71f, were established and their biological traits were explored. Methods: The proliferation, migration and invasion capabilities of HBE-Dp71d and HBE-Dp71f cells were evaluated by MTT, colony formation, transwell and scratch assay. Cell cycle and apoptosis induced by $\mathrm{H}_{2} \mathrm{O}_{2}$ were measured by flow cytometer. Co-IP was performed to prove the interaction between lamin B1, FAK and Dp71. Western blot was performed to detect lamin B1, FAK, ERK and Cyclin D expression in HBE-Dp71d and HBE-Dp71f cells. Results: HBE-Dp71d and HBE-Dp71f cells proliferated faster than their mock and blank controls; shortened their G0/G1 phase; enhanced their invasion and migration capabilities; reduced their apoptosis induced by $\mathrm{H}_{2} \mathrm{O}_{2}$. Co-IP proved Dp71 directly interacting with focal adhesion kinase (FAK) and lamin B1 in HBE cells. Increased lamin B1, FAK mRNA and protein expression, over activation of integrin/focal adhesion kinase/extracellular signal-regulated kinase (ERK)/cyclin D pathway were observed in HBE-Dp71d and HBE-Dp71f cells. Conclusions: Via increasing FAK in the cytoplasmic FAK-Dp71, lamin B1 of nucleus laminB1-Dp71 complex, HBE-Dp71d and HBE-Dp71f cells alter their proliferation, migration, invasion, cell cycle and apoptosis rate induced by $\mathrm{H}_{2} \mathrm{O}_{2}$.
\end{abstract}

S. Tan and J.Xie contribute equally to the work.

Sichuang Tan

and Jinliang Xie 


\section{Introduction}

Dystrophin is a 427-kDa rod-shaped protein, whose mutation causes Duchenne muscular dystrophy (DMD), an X-linked recessive disorder [1]. Dystrophin Dp71 consists of several C-terminal 70- to 75-kDa dystrophin isoforms named according to their molecular weight. Dystrophin Dp71 protein locates in all tissues except skeletal muscle. Dp71f and Dp71d, the two widely isoforms were found in the nucleus, cytoplasm and Golgi complex (GC) in hippocampal neuronal nuclei. It is suggested that Dp71 might play different roles from dystrophin based on its diverse tissue and cellular location $[2,3]$. Being the first product of the DMD gene detectable during development, relatively high Dp71 promoter activity was found associated with morphogenic events and terminal differentiation of several mouse tissues and organs, including the nervous systems, limb buds, lungs, blood vessels, eyes, inner ear, and nasal organs [4-6]. Based on the temporal and spatial expression profile, it is suggested that dystrophin Dp71 plays important and broad physiological roles in many organs that need further clarification.

Previous research revealed the participation of Dp71 in cell adhesion, water homeostasis, cell division, and nuclear architecture formation in PC12 cells [7]. As a ubiquitously expressed cytoskeleton protein, however, the biological roles Dp71 plays in cells other than PC12 remain unknown. Our current research identified increased Dp71 in rat pulmonary ischemia-reperfusion process. Enhanced Dp71 expression was found in HBE cells under $\mathrm{H}_{2} \mathrm{O}_{2}$ stimulation. Taking HBE as a usual cell model for the functional study of the lung epithelium, Dp71f and Dp71d over-expressing cell lines were set up to explore the influence of increased Dp71 isoforms. To further explore the biological function of Dp71, cell cycle, proliferation, invasion and migration capabilities, apoptosis induced by $\mathrm{H}_{2} \mathrm{O}_{2}$ were evaluated in HBE-Dp71d and HBE-Dp71f cell lines.

\section{Materials and Methods}

\section{Plasmids}

Plasmids expressing Dp71d and Dp71f isoforms were kind gifts from Dr Bulmaro Cisneros (Department of Genetics and Molecular biology, CINVESTAV-IPN, Mexico City). The Dp71d-control plasmid was constructed via re-circularizing with T4 DNA ligase, after the Dp71d coding region was removed by EcoRI. The Dp71f-control plasmid was constructed via religation of the pRC/CMV2 plasmid after the Dp71f coding region was removed by XbaI. For the Dp71d- pcDNA3.1-3xFlag-C:

Dp71a-F:

5' CTTGGTACCGAGCTCGGATCCGCCACCATGAGGGAACAGCTCAAAGG3',

Dp71a-R:5'TGCTGGATATCTGCAGAATTCCATTGTGTCCTCTCTCATTGGC3' were used to amplify the 1815 bp PCR product matches NM_004017.2(Dp71d).

For the Dp71f- pcDNA3.1-3xFlag-C plasmids,

Dp71b-F: 5' cttggtaccgagctcggatccGCCACCATGAGGGAACAGCTCAAAGG $\quad 3{ }^{\prime}, \quad$ Dp71b-R: 5' tgctggatatctgcagaattcTTCTGCTCCTTCTTCATCTGTC 3' were used to amplify the 1908 bp PCR product matches NM_004017.2 (Dp71f). All constructs were verified by restriction enzymes digest analysis and sequencing.

\section{Antibody}

Mouse anti-dystrophin monoclonal antibody; rabbit anti-dystrophin polyclonal antibody (Abcam); rabbit anti-lamin B1 polyclonal antibody, rabbit anti-FAK polyclonal antibody, p-FAK polyclonal antibody; rabbit anti-ERK polyclonal antibody, p-ERK polyclonal antibody; rabbit anti cyclin D polyclonal antibody; (Bioworld Technology, Inc), mouse anti-Flag antibody, mouse antiglyceraldehyde-3-phosphate dehydrogenase (GAPDH) monoclonal antibody (Sigma); peroxidase-conjugated anti-mouse and anti-rabbit IgG, titric-conjugated anti-mouse and anti-rabbit IgG (Boster Biological Technology, Shanghai, China) were used in the experiments. 


\section{Cellular Physiology Cell Physiol Biochem 2017;43:2022-2036 \begin{tabular}{l|l|l} 
DOI: 10.1159/000484181 & $\begin{array}{l}\text { O 2017 The Author(s). Published by S. Karger AG, Basel } \\
\text { www.karger.com/cpb }\end{array}$
\end{tabular}}

Tan et al.: Dp71 Enhanced HBE Cells

Cell culture and generation of stable transfectans

HBE cells were grown in Dulbecco's modified Eagle medium (DMEM) (Gibco), supplemented with $10 \%$ fetal bovine serum, and maintained at $37^{\circ} \mathrm{C}$ in a humidified incubator with a $5 \% \mathrm{CO}_{2}$ atmosphere. For stable transfection, HBE grown in $25-\mathrm{cm}^{2}$ dishes, transfected with 5 ug of plasmid DNA pre-mixed with 300 ul serum-free DMEM (Gibco), 10 ul Lipofectamine ${ }^{\mathrm{TM}} 2000$ Transfection Reagent (Invitrogen) was added in the previous mixture, incubated at room temperature for $5 \mathrm{~min}$, added into the serum free DMEM. After a 4 hours incubation period, the transfection mixture was removed and cells were maintained in DMEM supplemented with sera. Selection of stable transfectans was initiated $48 \mathrm{~h}$ after transfection with $600 \mathrm{ug} /$ $\mathrm{ml}$ of G418 (Invitrogen), a neomycin analog.

\section{Isolation of cell extracts and Western blot analysis}

Isolation of cell extracts and western blot analysis were the same as described previously [8]. In brief, 60 ug of whole cell protein extracts were denatured with $5 \times$ sodium dodecyl sulfate (SDS) loading buffer and proteins were denatured by boiling for $3 \mathrm{~min}$. Lysates were then separated by 10\% SDS-PAGE and electrotransferred to nitrocellulose membranes. Membranes were incubated for $1 \mathrm{~h}$ in TBS-T (150 mM NaCl, $10 \mathrm{mM}$ Tris-HCl, $\mathrm{pH}$ 8, 0.05\% Tween20) containing 6\% low-fat dried milk followed by being incubated overnight with the corresponding primary antibody. After three washes with TBS-T, membranes were incubated with the appropriate horseradish peroxidase-conjugated secondary antibody and developed using the ECL Western blotting analysis system (Amersham-Pharmacia).

\section{Quantitative Real-time Polymerase Chain Reaction (QRT-PCR) and RT-PCR}

Total RNA was prepared using Trizol (Invitrogen) according to the manufacturer's protocol. cDNAs from all samples were synthesized from $1.0 \mu \mathrm{g}$ of total RNA by PrimeScript ${ }^{\circledR}$ 1st strand cDNA Synthesis Kit (TaKaRa Bio, Siga, Japan). The synthesized cDNA samples were subjected to qPCR using SYBR® Green Quantitative PCR kit (TaKaRa Bio, Siga, Japan). Amplification was carried out in a total volume of $20 \mu \mathrm{L}$ for 40 cycles of $15 \mathrm{~s}$ at $95^{\circ} \mathrm{C} 20 \mathrm{~s}$ at $60^{\circ} \mathrm{C}$ and $30 \mathrm{~s}$ at $72^{\circ} \mathrm{C}$ Samples were run in triplicate and their relative expression was determined by normalizing expression of each target to 18S. All experiments were conducted 3 times using BioRad CFX96 C1000 System (BioRad, Hercules, CA).The primers were designed using Primer3 online software. $\Delta \Delta \mathrm{C}_{\mathrm{T}}$ calculations were made to normalize signal versus $18 \mathrm{~S}$ rRNA as the control.The following primers were used and they produced a 173bp PCR product for FAK, 146bp PCR product for Lamin B1, and 181bp PCR product for 18s. They are:

\section{LMNB1 (NM_005573) F: 5'TCCAGGAGAAGGAGGAGCTG3', \\ R: 5'GGTCTCGTAGAGCGCCTTG3'; \\ FAK (L13616.1) F: 5' TCCCCAGAGCTCCTCAAGAA 3', \\ R: 5' TACTCGCTCCATTGCACCAG3'; \\ Human 18S (NM_022551) F: 5' AAATAGCCTTTGCCATCACTGCC 3', \\ R: 5' GTTCAAGAACCAGTCTGGGATC3'}

A revised PCR was performed for the characterization of Dp71d and Dp71f in HBE as described [9]: Nested Dp71F (5' agccagcctc gtagtcctgc ccag 3') and Nested Dp71R( 5'agttgttgagt tgctccatca cc 3') were used for the first round PCR of synthesized cDNA samples. Then 1ul PCR product was used as the template for the $2^{\text {nd }}$ PCR with Dp71 77F2 (5'CTCCCCAggACACAAgCACAg 3') and Dp79R (5' TTATTCTgCTCCTTCTTCATCTgTCATgACTg 3') as the primers. The primers match NM_004017.2 (Dp71a) and NM_004016.2 (Dp71b) perfectly and the predicted $2^{\text {nd }}$ PCR produced products are $202 \mathrm{bp}$ (with exon 78 ) and $170 \mathrm{bp}$ (without exon $78 \Delta 78$ ), respectively.

\section{Immunofluorescence and confocal microscopy analysis}

The immunofluorescence and confocal microscopy analysis of HBE, HBE transfected with Dp71d- pcDNA3.1-3xFlag-C, Dp71f- pcDNA3.1-3xFlag-C and their control plasmids were as follows. After the HBE Cells cultured on glass coverslips were treated as described previously, they were incubated overnight at ${ }^{\circ} \mathrm{C}$ with the mouse anti-Flag antibody. The day after, cells were washed in PBS and incubated with a titric-conjugated secondary anti-mouse IgG antibody. Cells were incubated for $10 \mathrm{~min}$ at $37^{\circ} \mathrm{C}$ with $1 \mathrm{mg} / \mathrm{ml} \mathrm{49,6-diamidino-2-phenylindole} \mathrm{(DAPI)} \mathrm{for} \mathrm{counterstaining,} \mathrm{after}$ washing, coverslips were mounted on microscope slides with VectaShield (Vector Laboratories, Inc., Burlingame, CA, USA) and analyzed in a confocal and multiphoton microscope (TCS-SP5, Leica 


\section{Cellular Physiology Cell Physiol Biochem 2017;43:2022-2036 \begin{tabular}{l|l|l} 
DOI: 10.1159/000484181 & $\begin{array}{l}\text { O 2017 The Author(s). Published by S. Karger AG, Basel } \\
\text { www.karger.com/cpb }\end{array}$ \\
\hline
\end{tabular} \\ Tan et al.: Dp71 Enhanced HBE Cells}

Microsystems, Heidelberg, Germany), using an oil immersion 636 objective. Co-localization of FITC and DAPI staining was analyzed in single optical sections obtained for two channels throughout the $\mathrm{Z}$ axis. Cell cycle analysis

Cells from each group were plated in a six-well plate at a concentration of $1 \times 10^{5} \mathrm{cells} / \mathrm{ml}$, and they were collected after $48 \mathrm{~h}$ incubation. Cells were washed with pre-cooling PBS for 2 times before they were fixed in $70 \%$ ethanol and stained with propidium iodide. The DNA content was analyzed with an Epics Profile II flow cytometer (Beckman Coulter) and analysis with Multicycle software (Phoenix Flow Systems). All experiments were repeated three times.

\section{Plate colony formation assay}

Cells in the logarithmic growth phase of each group were digested and the single cell suspension was inoculated into 6 well plates. $2 \mathrm{ml}$ culture was plated in each well; the concentration of cell was

$5 \times 10 / \mathrm{ml}$. They were cultured at $37^{\circ} \mathrm{C}$ with $5 \% \mathrm{CO} 2$ and saturated humidity conditions until visible clone cell clusters with naked eye appeared in culture dish, then the culture was terminated. Cells were washed with PBS carefully for 2 times. Each well was added into $800 \mu \mathrm{l}$ methanol and fixed for 30 minutes. Then $1 \mathrm{ml}$ toluidine blue staining solution was added in each well and stained for 30 minutes. Each plate was washed with water slowly and dried in air. The visible clones were counted with image analysis software and the percentage of clone formation was calculated.

\section{Cell viability assay (MTT assay)}

Cells were harvested during logarithmic growth phase and seeded in 96-well plates (Corning, Costar) at a density of $1000 \mathrm{cell} / \mathrm{mL}$, freshly prepared sterilized MTT (Sigma-Aldrich, Inc.) was added to each well at each time point to a final concentration of $0.5 \mathrm{mg} / \mathrm{ml}$ and plates were further incubated for $4 \mathrm{~h}$ at $37^{\circ} \mathrm{C}$. Cells were centrifuged for $5 \mathrm{~min}$ at 1, $500 \mathrm{rpm}$ and the supernatant was discarded; $180 \mathrm{ml}$ of dimethylsulfoxide was then added and plates were shaken until no particulate matter was visible. Absorbance was measured on a Molecular Devices Spectra Max Plus384 microplate reader (Molecular Devices, Sunnyvale, CA, USA) with a wavelength of $570 \mathrm{~nm}$. The cell growth curve was drawn by plotting the mean cell number of each point against the cell culture time.

\section{Invasion assay}

The cell invasion assay was performed using the BD BioCoat ${ }^{\mathrm{TM}}$ 24-Multiwell Invasion System precoated with BD Matrige ${ }^{\mathrm{TM}}$ Matrix (BD Biosciences, USA). $1 \times 10^{6}$ cells in serum-free medium containing $0.1 \%$ bovine serum albumin were placed into the upper chamber. The lower chamber was filled with $500 \mathrm{ml}$ of media containing $10 \%$ FBS. After a 24 -hour incubation at $37^{\circ} \mathrm{C}$, non-invading cells were removed from upper side of transwell membrane filter inserts using a cotton-tipped swab. Invaded cells on the lower side were stained and the absorbance was read at $560 \mathrm{~nm}$. For each experimental group, the invasion assay was performed in triplicate.

\section{In vitro wound healing assay (scratching test)}

Wound healing assay was performed briefly as follows: HBE-Dp71d, HBE-Dp71f and respective control cell groups were grown in six-well plates until confluence. A scrape was made through the confluent monolayer with a sterile plastic pipette tip of $1 \mathrm{~mm}$ diameter. Afterwards, the dishes were washed twice with PBS and incubated at $37^{\circ} \mathrm{C}$ in fresh DMEM complemented with $10 \%$ fetal bovine serum. At the bottom side of each dish, three arbitrary places were marked where the width of the wound was measured with an inverted microscope (objective $\mathrm{x} 10$ ). Representative images of cells migrating into the wounds were captured in the same wounded region using an inverted microscope at different time points. Would closure rate $=(0 \mathrm{~h}$ scratch width $-48 \mathrm{~h}$ scratch width $) / 0$ h scratch width $\times 100 \%$.

\section{Apoptosis induced by $\mathrm{H}_{2} \mathrm{O}_{2}$}

The HBE, HBE-Dp71f, Dp71f control, HBE-Dp71d, Dp71d control cell lines grown in six well plates until $70 \%$ confluence reached. Then the medium was replaced by DMEM contained $200 \mu \mathrm{M} \mathrm{H}_{2} \mathrm{O}_{2}$ (Sigma, USA), and the cells were cultured for another 16 hours. The cells were collected and the apoptosis assays were the same as described previously [10]. 


\section{Cellular Physiology Cell Physiol Biochem 2017;43:2022-2036

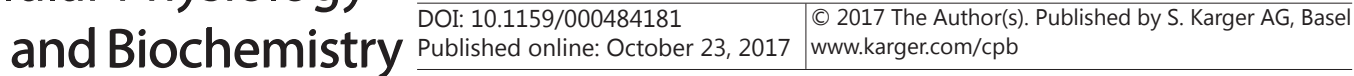

Fig. 1. Characterization of HBE-Dp71d and HBEDp71f cells. (A). Characterization of Dp71 isoforms in HBE. RT-PCR identified presence of exon 78 (+78) Dp71d or spliced out $(\Delta 78)$ Dp71f isoforms in HBE. Lane 1 Dp71 upper band; lane 2 Dp71 lower band. The size of the PCR products is indicated on the right. Molecular size in base pairs is indicated on the left. (B). Expression and subcellular distribution of endogenous Dp71 isoforms in HBE cells. As can be seen in the confocal image, Dp71 was expressed in both nucleus and cytoplasm in HBE cells. (C). Immunoblot analysis of Dp71 expression in stably transfected HBE clones. (D). Statistical analysis of Dp71 expression in five HBE cell lines. The increased Dp71 protein in HBE-Dp71d and HBE-Dp71f cells are of statistically significance. (E) Immunofluorescence analysis of exogenous expressed Dp71 in HBE. Dp71d- pcDNA3.1-3xFlag-


E.



Dp71d-pcDNA3.1

Dp71f-peDNA3.1
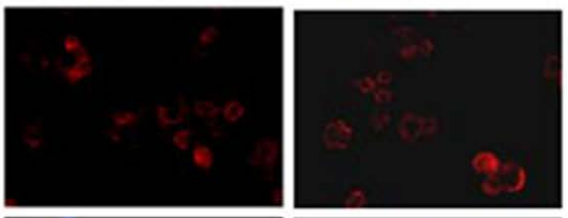

DAPI
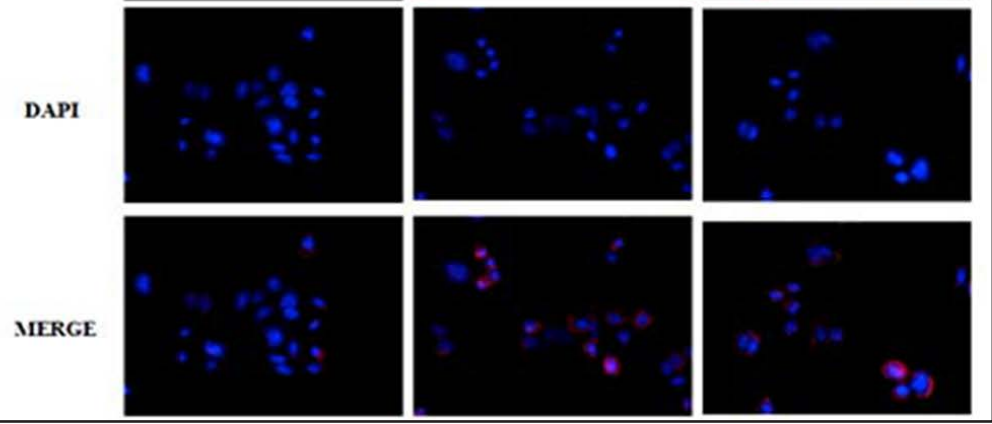

C, Dp71f- pcDNA3.1-

3xFlag-C, and empty pcDNA3.1-3xFlag-C plasmids were transfected into HBE cells. As indicted in Fig. E, exogenous Dp71d and Dp71f displayed both cytoplasmic and nucleus locations.

\section{Statistical analyses}

All assays were repeated 3 times to ensure reproducibility. For comparisons of the 3 assays and those between groups, ANOVA and Student's t-test were made respectively. All tests were made using SPSS 17.5. Results are displayed as the means \pm SD. $\mathrm{P}<0.05$ was considered to indicate a statistically significant difference.

\section{Results}

\section{Characterization of Dp71 spliced isoforms in HBE}

Dp71d and Dp71f were proved to be the two most dominant spliced isoforms in HeLa, C2C12, N1E-115 and PC12 cell. Using primers flanking exon 78, RT-PCR identified that both Dp71f and Dp71d are present in HBE cells (Fig. 1A). Confocal analysis with Dp71 specific antibody (ab15277) identified the nucleus and cytoplasmic location of Dp71 in HBE (Fig. 1B). Our characterization of Dp71 in HBE proved the expression of Dp71d and Dp71f in another cell line.

\section{KARGER}




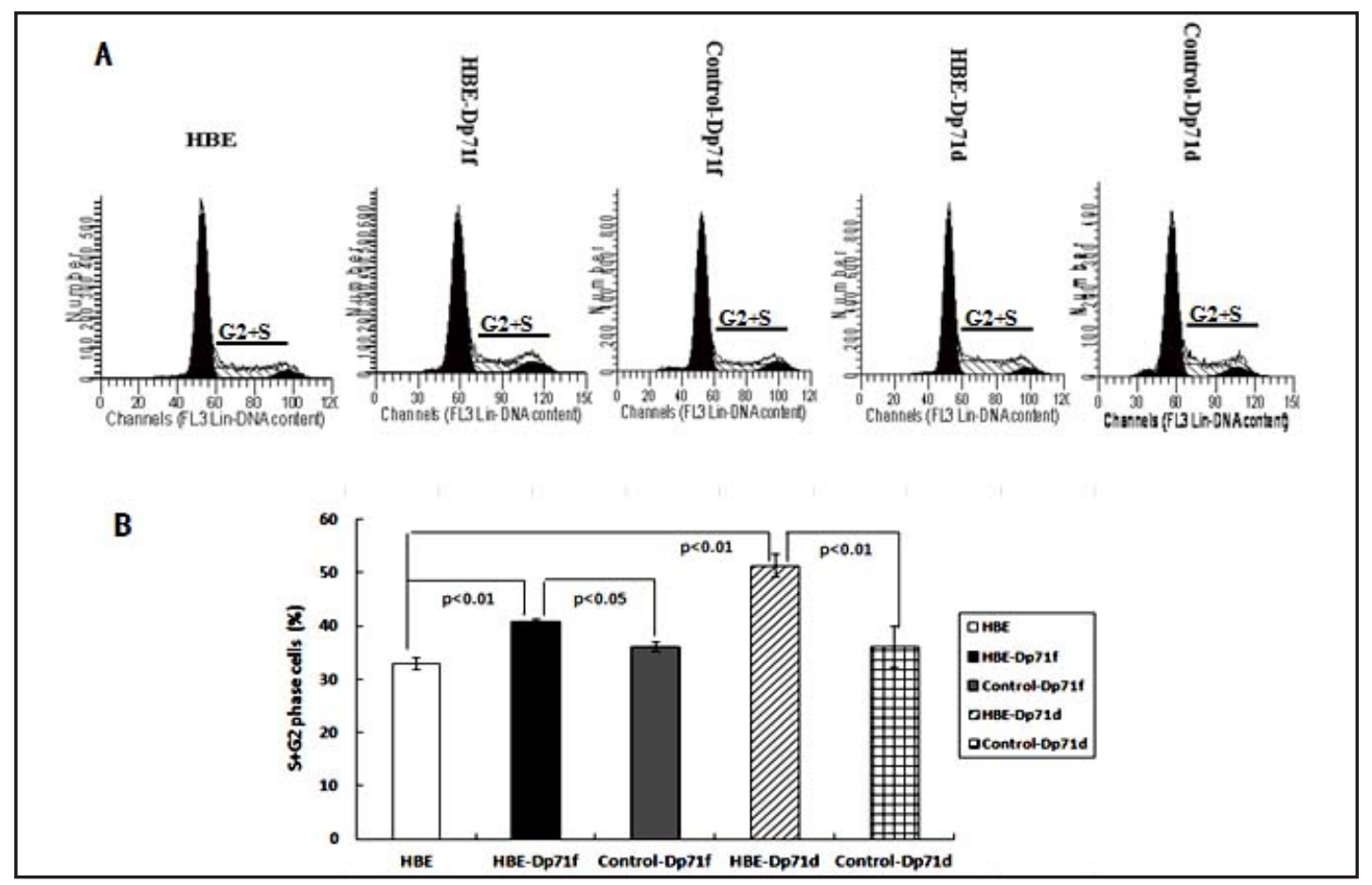

Fig. 2. HBE-Dp71d and HBE-Dp71f cells change their cell cycle. Cycle analysis revealed over-expressing of Dp71d and Dp71f can increase the cell distribution in G2/S phase.

\section{Establishment of Dp71 over-expression HBE cell lines}

To begin our assessment of Dp71 protein function in HBE, we established cell lines over-expressing two Dp71 isoforms (Dp71f and Dp71d) and the respective control vectors in normal human bronchial epithelium (HBE). Since there were no commercially available antibodies which could differentiate Dp71f and Dp71d, anti-dystrophin antibodies from Abcam (ab15277, ab7124), which could recognize both isoforms, were used in our research. Western blot was performed to analyze the Dp71 expression in each stable transfectant (namely HBE-Dp71f for Dp71f over-expressing cell line, Dp71f control for control plasmid, HBE-Dp71d for Dp71d over-expressing cell line, Dp71d control for control plasmid). As indicated in Fig. 1C, each stably transfected cell line indicated much higher Dp71 expression than the vector control and blank cells, and the differences were statistically different (Fig. 1D).

In order to clarify the subcellular location of exogenously expressed Dp71 isoforms, Dp71d-pcDNA3.1-3xFlag-C and Dp71f-pcDNA3.1-3xFlag-C plasmids were constructed and transfected into HBE cells. Although Dp71f was proposed to display a cytoplasm and cell periphery, yet it was found that of Dp71d was concentrated in the nucleus, and that a dissipated cytoplasmic and nucleus expression of exogenous Dp71d and Dp71f were observed in both Dp71 over-expressing HBE cells. The immunofluorescence photos showed that the exogenously increased Dp71d and Dp71f protein displayed a similar cytoplasmic and nucleus subcellular distribution (Fig. 1E).

\section{HBE-Dp71d and HBE-Dp71f cells shorten their G0/G1 phase}

Previous Dp71 research revealed that Dp71 was a component of the multi-protein apparatus of mitosis and cytokinesis in PC12 cells. Knocking down Dp71 expression resulted in an impaired cell cycle and delay in G0/G1 transition in PC12 cells. Flow cytometry was used to analyze respective cell cycle in HBE-Dp71d and HBE-Dp71f cell lines. As revealed in Fig. 2, HBE-Dp71d and HBE-Dp71f cells displayed an increased cell distribution in S+G2 phase and a concomitant reduction in G1 phase (Fig. 2). The shortened G1 phase in both 




Fig. 3. HBE-Dp71d and HBE-Dp71f cells demonstrated increased proliferation capabilities and plate colony formation A. MTT assay demonstrated that HBE-Dp71f and HBE-Dp71d cells can proliferate faster than their vector control and parental cells. B. Plate clone formation assay demonstrated that HBE-Dp71f and HBE-Dp71d cells can form more clones than the mock and parental cells. C. Statistical analysis of the plate clone formation assay, the differences of the clone formation efficiency are significant.

HBE-Dp71f and HBE-Dp71d cells proved that up-regulation of Dp71d and Dp71f in HBE cells can alter their cell cycles.

HBE-Dp71d and HBE-Dp71f cells demonstrated increased proliferation and plate colony formation

MTT assay was performed to evaluate the proliferation rates of the stably transfected HBE-Dp71d and HBE-Dp71f clones during a period of 3 days. Fig. 3 displayed both Dp71f cells and Dp71d cells grew much faster than the parental and control vector-transfected cells, which suggested that increasing the two of the Dp71 isoforms could accelerate the growth of HBE. In the plate colony formation assays of the five HBE cell lines, HBE-Dp71d and HBE-Dp71f clones showed phenotypes of higher colony forming efficiency than their respective vector control and parental cells. The differences between the five HBE cells were statistically significant (Fig. 3B \&3C). All the data revealed that enhanced Dp71 could increase their proliferation capabilities.

HBE-Dp71d and HBE-Dp71f cells demonstrate increased migration and invasion capabilities

The scratch assay was performed to assess whether the cell motility of HBE-Dp71f and HBE-Dp71d cells changed. As shown in the Fig. 4A, over-expressing of both Dp71f and KARGER 


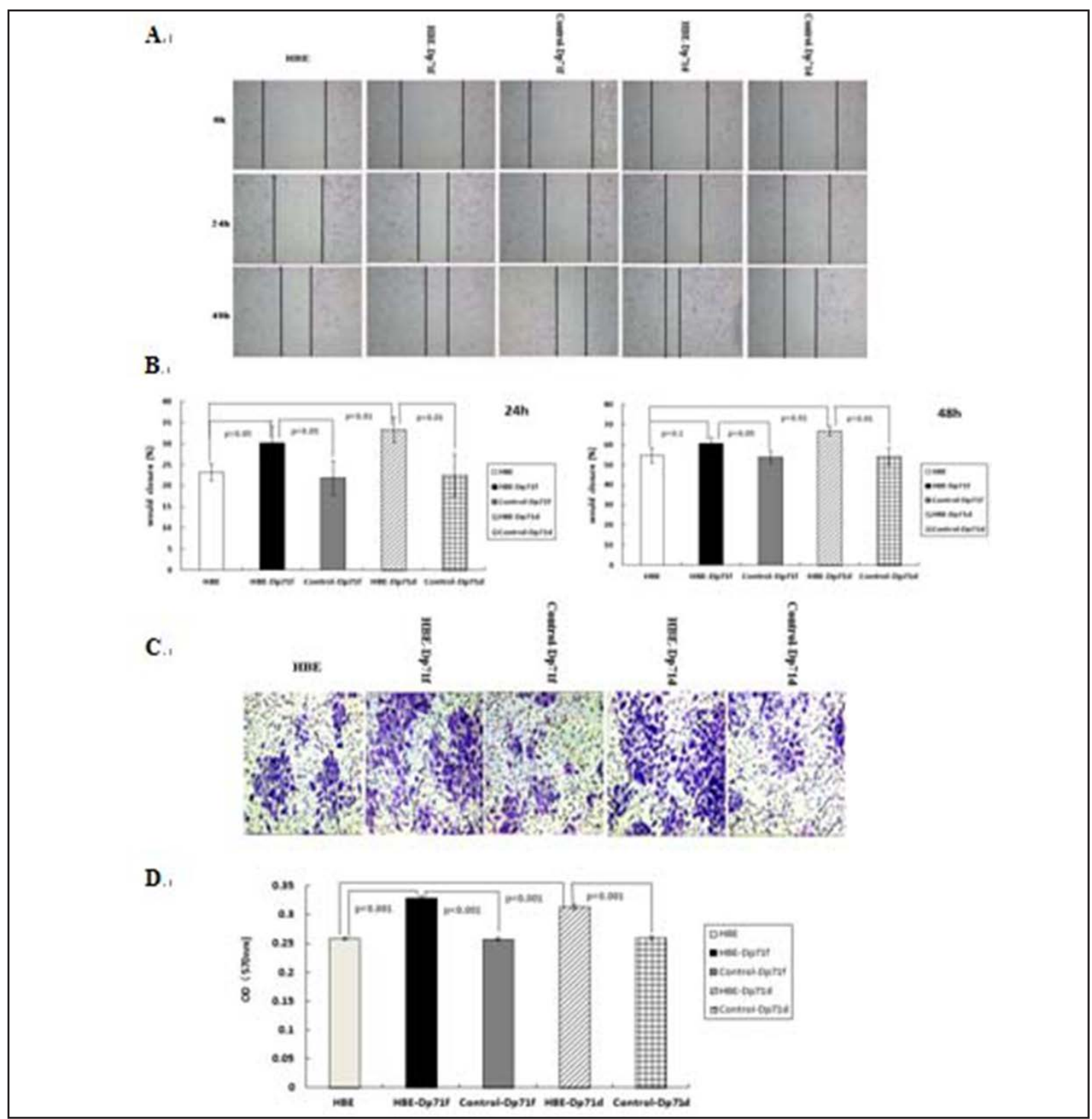

Fig. 4. HBE-Dp71d and HBE-Dp71f cells displayed enhanced migration and invasion capabilities. (A) Would healing assay display the HBE-Dp71f and HBE-Dp71d cell lines increased their migration capabilities. (B) Transwell assay revealed the increased invasive capabilities of HBE-Dp71f and HBE-Dp71d cell lines. (C) Statistical analysis of would healing assay of five HBE cell lines at 48 hours, the difference were significant. (D) Statistical analysis of average OD value at $560 \mathrm{~nm}$, of the five HBE cell lines, the differences were significant.

Dp71d isoforms can accelerate wound closure speed of HBE-Dp71f and HBE-Dp71d cells at $48 \mathrm{~h}$. Statistical analyses showed that differences of the relative invasion of the five HBE cells were significant (Fig. 4C), indicating that the two of the Dp71 isoforms can enhance the migration capabilities of HBE cells.

Invasion capabilities of HBE-Dp71f and HBE-Dp71d cells were measured by HTS multiwell insert system. The results indicated that the numbers of HBE-Dp71f and HBE-Dp71d cells passing through the Matrigel were much larger compared with the control-Dp71d, control-Dp71f and parental cells (Fig. 4B). Statistical analyses showed that differences of the relative invasion of the five HBE cells were significant (Fig. 4D). Together with scratch assay results, our data suggested that increasing the two of Dp71 isoforms can significantly improve HBE migration and invasion.

\section{KARGER}






Fig. 5. Decreased apoptosis rate induced by $\mathrm{H}_{2} \mathrm{O}_{2}$ in HBE-Dp71d and HBE-Dp71f cells (A) The five representative dot plots of the HBE cells, HBE-Dp71d cells, Co ntrol-Dp71d cells, HBE-Dp71f cells, ControlDp71f cells exposed to $\mathrm{H}_{2} \mathrm{O}_{2}$ contained medium for 16 hours, the apoptosis was determined by Annexin V/ PI staining and flow cytometric analysis. Similar results were observed in 3 independent experiments. (B) Statistical analysis of the apoptosis rate induced by $\mathrm{H}_{2} \mathrm{O}_{2}$, the differences were statistically significant.

HBE-Dp71d and HBE-Dp71f cells displayed reduced apoptosis induced by $\mathrm{H}_{2} \mathrm{O}_{2}$

In order to see whether the alteration of Dp71 can change the apoptosis process of $\mathrm{HBE}$, the five $\mathrm{HBE}$ cell lines were treated with culture medium contained $200 \mu \mathrm{M} \mathrm{H}_{2} \mathrm{O}_{2}$. As shown in dot plot images generated by FACS analysis of cells stained with Annexin V (Fig. 5A), HBE-Dp71f cells $(8.183 \pm 1.17)$ displayed 10\% less apoptosis compared with HBE cells (18.67 \pm 3.614$), 11 \%$ less apoptosis compared with Control-Dp71f cells $(20.05 \pm 3.713)$. HBEDp71d cells (6.74 \pm 1.933$)$, displayed $12 \%$ less apoptosis compared with HBE cells, $12 \%$ less apoptosis compared with Control-Dp71d cells $(18.55 \pm 1.057$, Fig. 5A). The differences were statistically significant (Fig. 5B).

\section{Dp71 associated with FAK in HBE cells}

Dp71 was found associating with most of the $\beta 1$-integrin complex components ( $\beta 1$ integrin, FAK, $\alpha$-actinin, talin and actin) in PC12 cells [10]. Co-Ip assays were performed to detect the association between FAK and Dp71 in HBE cells. Total protein extracts from HBE cells were immunoprecipitated with anti-FAK or anti-Dp71 antibody, precipitated proteins were analyzed by immunoblotting with antibodies directed specifically to either FAK or Dp71. Fig. 6A showed that FAK was pulled down together with Dp71 by the anti-Dp71 antibody in HBE cells, whereas none of these two proteins was recovered when an irrelevant antibody (IgG) was used for immunoprecipitation, which establishing the specificity of the assays. In Fig. 6B, compared with the non-specific IgG, Dp71 was pulled down together with rabbit anti-FAK-antibody. Co-ip with each specific antibody displayed association between Dp71 and FAK in HBE, which proved that FAK is an indispensable component of the cytoplasmic DAPCs.

HBE-Dp71d and HBE-Dp71f cells displayed increased FAK/ERK/cyclinD pathway expression and over-activation

Real time RT-PCR was also performed to detect FAK mRNA expression, as indicated in Fig. 6C, significant FAK mRNA can be detected in both HBE-Dp71d and HBE-Dp71f cells, 


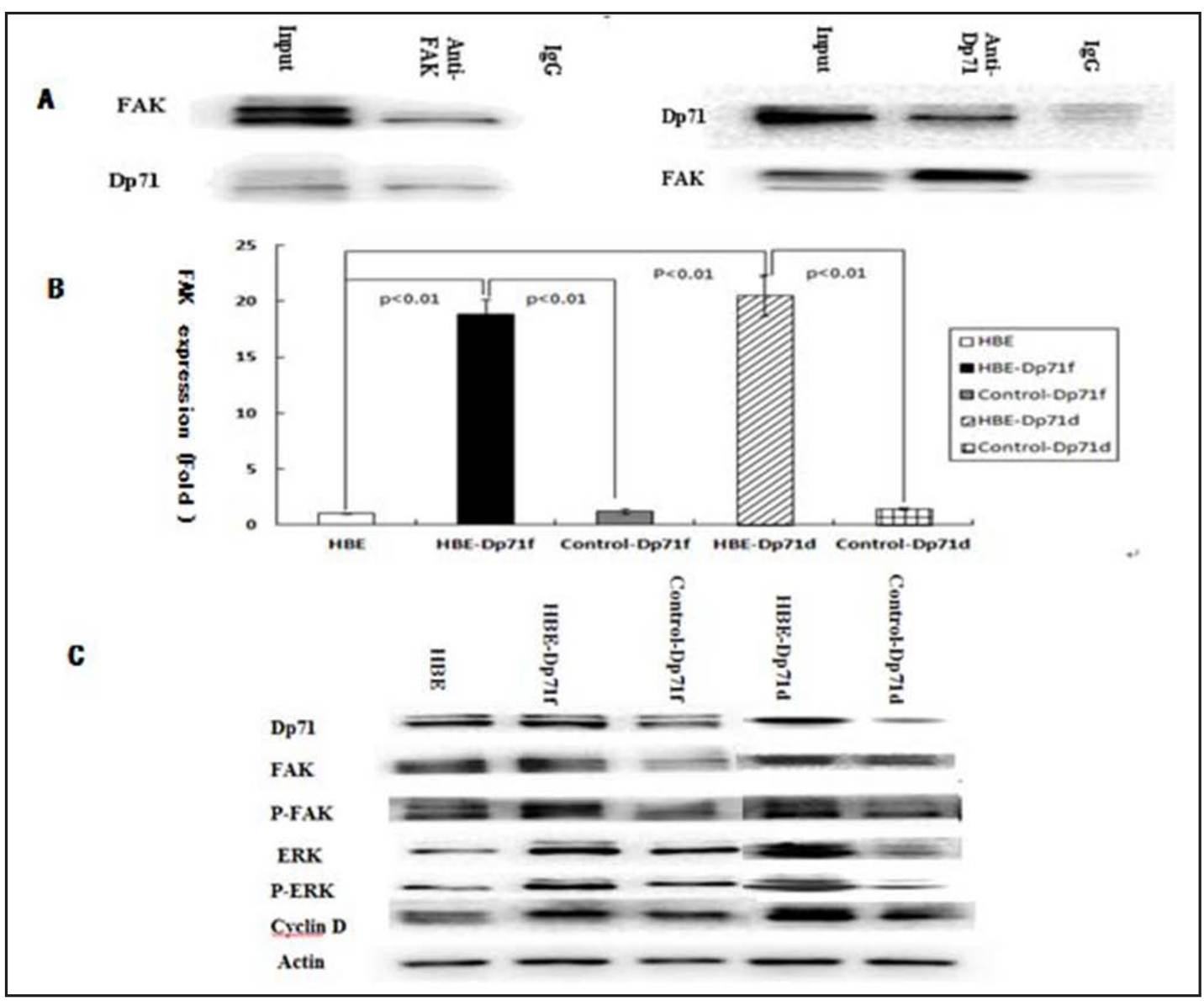

Fig. 6. Increased FAK mRNA and protein in HBE-Dp71d and HBE-Dp71f cells (A) Dp71 associates with FAK in HBE cells. Whole cell extracts from HBE cells were immunoprecipitated with a rabbit anti-FAK antibody (left), rabbit anti-Dp71 antibody (right), an irrelevant antibody (IgG0) was used as control. (B) Real time RTPCR analysis of FAK mRNA in five HBE cells, significantly higher FAK mRNA expression was detected in HBEDp71f and HBE-Dp71d cells. (C) Western blot was performed to analyze the relative FAK,p-FAK, ERK, p-ERK, cyclin D protein expression in the five HBE cell lines. As displayed in the immune-blot, higher expressions of FAK,p-FAK, ERK,p-ERK, cyclin D were detected in HBE-Dp71f and HBE-Dp71d cells.

which means that up-regulate Dp71 can enhance FAK expression from the transcription level.

Western blot was performed to measure the FAK protein in HBE-Dp71d and HBE-Dp71f cells. As indicated in Fig. 6D, significantly increased FAK protein was observed in HBE-Dp71d and HBE-Dp71f cells, further immunoblot assay identified overactivation of FAK. Western blot was used to detect the ERK and p-ERK expression in HBE-Dp71d and HBE-Dp71f cells, as can be seen in Fig. 7B, increased ERK and p-ERK expression occurred in both Dp71 over-expressing HBE cells. Cyclin D is a key mediator of G1/S phase transition activated by p-ERK. Western blot proved the increased expression of down stream molecule cyclin D. In summary, enhanced Dp71d and Dp71f lead to the increased expression and over activation of FAK/ERK/ cyclin D pathway.

\section{Dp71 associate with nucleus lamin B1 in HBE cells}

Lamin B1 is one of the nucleus cytoskeleton proteins interacting with Dp71 in PC12 cells. In our further characterization of the nuclues DAPCs in HBE, co-ip was performed to verify the interaction between lamin B1 and Dp71. Anti-lamin B1 and anti-Dp71 antibody were used for immunoprecipitation in the HBE whole protein lysate. In Fig. 7A, lamin B1 


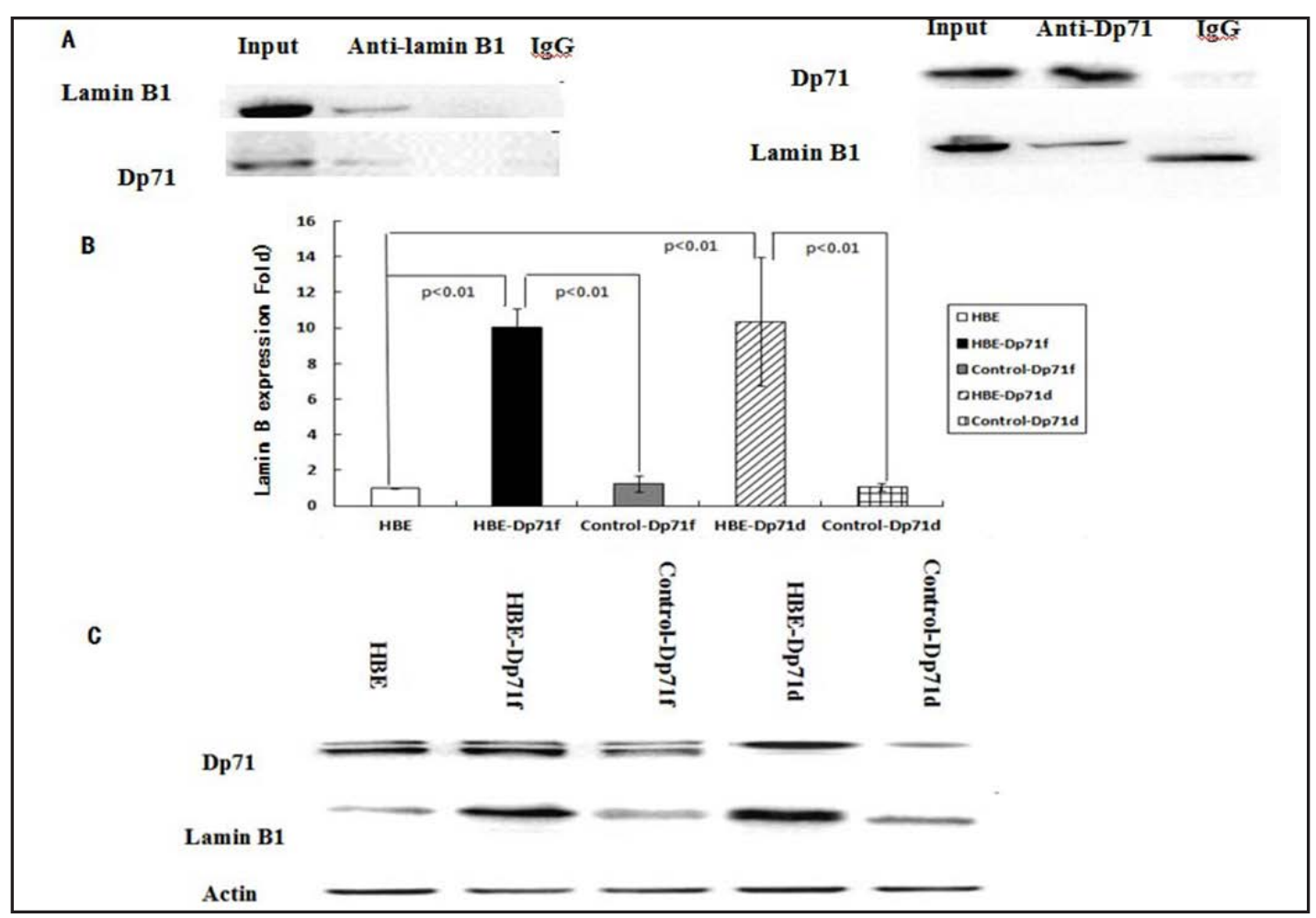

Fig. 7. Increased lamin B1 mRNA and protein in HBE-Dp71d and HBE-Dp71f cells(A) Dp71 associates with lamin B1 in HBE cells. Whole cell extracts from HBE cells were immunoprecipitated with a rabbit anti-lamin B1 antibody (left), rabbit anti-Dp71 antibody (right), an irrelevant antibody (IgG0) was used as control. Immunoprecipitated proteins were analyzed by western blotting with mouse anti-lamin B1 and anti-Dp71 antibodies. Association between Dp71 and lamin B1 was observed in HBE cells. (B) Real time RT-PCR analysis of lamin B1 mRNA in five HBE cells. Higher lamin B1 mRNA expression was detected in HBE-Dp71f and HBE-Dp71d cells. (C) Western blot showed higher lamin B1 expressions in HBE-Dp71f and HBE-Dp71d cells.

was pulled down together with rabbit anti-Dp71 antibody while IgG failed to precipitate these two proteins. Fig. 7A showed that Dp71 was pulled down together with lamin B1 by the anti-lamin B1 antibody in HBE cells, whereas none of these two proteins was recovered when an irrelevant IgG was used for immunoprecipitation. Co-immunoprecipitation with each specific antibody showed the direct association between Dp71 and lamin B1, which proved the existence of nuclear Dp71-lamin B1 protein complex in HBE cells.

\section{HBE-Dp71d and HBE-Dp71f clones demonstrated increased lamin B1 expression}

Previous research identified decreased lamin B1 expression in Dp71 knocking down PC12 and A549 cells. Real time RT-PCR and western blot were used to detect lamin B1 mRNA and protein expression in HBE-Dp71d and HBE-Dp71f cells. Significantly increased lamin B1 mRNA and protein were observed in HBE-Dp71f and HBE-Dp71d cells (Fig. 7B and 7C), which means that increased Dp71d and Dp71f isoforms can both enhance lamin B1 expression from both transcription and protein level in HBE.

\section{Discussion}

Functioning as a ubiquitously expressed protein, Dp71 might participate in basic functions that are common to all tissues and cell types. Previous research of Dp71 in PC12 cells revealed its participation of diverse biological function such as cell adhesion, cell 
division and nuclear architecture. Dp71 was also found to play a tumor suppressive role in gastric cancer [11-15]. The basic biological function of Dp71, however, needs further exploration in cells other than PC12 cells.

We initiated the research of Dp71 based on our previous research of HBE under oxidative stress. RT-PCR identified the existence of Dp71f and Dp71d isoforms in HBE, immunofluorescence proved the nucleus and cytoplasmic Dp71 location in HBE. Via setting up HBE cell lines over-expressing exogenous Dp71d and Dp71f, MTT assay and plate colony formation proved that both HBE-Dp71d and HBE-Dp71f cells proliferated faster than the mock cells and parental cells. Transwell and wound healing experiments demonstrated that HBE-Dp71f and HBE-Dp71d cells increased their invasion and migration capabilities. Also, cell cycle analysis showed that both HBE-Dp71f and HBE-Dp71d cells changed their cell cycle via shortening their G0/G1 phases.

Via associating with dystrophin associated protein complex, dystrophin was proved to play important roles in various organs $[16,17]$. Via up-regulation of FAK in the cytoplasmic DAPCs and lamin B1 in the nuclues from the transcription level, the HBE-Dp71d and HBEDp71f cells altered their biological traits in several different aspects. Focal adhesion kinase (FAK) is a broadly expressed tyrosine kinase implicated in cellular functions such as migration, growth, apoptosis and survival [18-20]. In PC12 cells, FAK was proved to be a cytoplasmic DAPC which interact with Dp71 [10]. In HBE cells, the interaction between FAK and Dp71 was also verified. As an important component of focal adhesion complexes, FAK lies within and coordinates signals through the focal adhesion multi-protein complex. Upon activation by FAK, ERK translocates from the cytoplasm to the nucleus, where ERK phosphorylates various transcription factors regulating cell cycle progression [21, 22]. For the first time, increased FAK and p-FAK were observed in Dp71 over expression cells. Enhanced ERK and p-ERK were also displayed in HBE-Dp71d and HBE-Dp71f cells. Functioning as an important anti-apoptotic and cell growth pathway, enhanced and over activation of FAK-ERK in HBEDp71f and HBE-Dp71d cells can explain their proliferation, migration, invasion alterations. Functioning as a key mediator of G1/S phase transition, cyclin D can be activated by ERK to form an activated cyclin D/CDK4/6 complex, thereby driving entry of the cell into the next phase of the cell cycle [23-26]. The increased cyclin D in HBE-Dp71f and HBE-Dp71d cells can explain their altered cell cycle and increased proliferation rate.

Working as a nuclear scaffolding protein, Dp71 was found to be critical for proper localization and stability of nuclear envelope. Significant reduction of nuclear laminin, agrin and DAPs were observed in mdx mice brain. In PC12 cells, Dp71 was identified as a component of the mitotic spindle and cytokinesis multi-protein apparatuses. The Dp71lamin B1 complex was proved to modulate the cell division cycle in PC12 cells [14]. Via reducing the nucleus lamin B1 protein, reduced Dp71 was proposed to play an important tumorigenesis role in cancer development [13]. In our further characterization of nucleus Dp71 protein complex in HBE-Dp71f and HBE-Dp71d cells, lamin B1 was found to be an indispensable part of Dp71 nucleus protein complex (Fig. 8). Research proved the key function of lamin B1 in maintaining integrity of the nuclear envelope and ensuring normal cell cycle progression [27]. Enhanced lamin B1 protein in the Dp71 nucleus complex may also play an important role in altering the HBE biological characters.

In our further characterization of HBE-Dp71f and HBE-Dp71d cells, FAK and lamin B1 were found to be enhanced from transcription level, which suggests that some basic transcription mechanism has been changed in these Dp71 over-expression cells. How can Dp71 regulate these two molecules from both mRNA and protein level? There still remain some unknown Dp71 interacted nucleus proteins which can participate in the basic transcription and translation machinery. Techniques such as immunoprecipitation-Mass spectrometry analyses can be used to identify other nuclear Dp71 interaction proteins. The NE (nuclear envelope) membrane proteome Dp71 participates may provide us with more clues for explanations of the altered biological traits of HBE-Dp71f and HBE-Dp71d cells.

Quite a few publications proved the unique cytoplasm location of Dp71f and predominant nucleus location of Dp71d in PC12 cells [28, 29], however, research on 
Fig. 8. Hypothetic model showing the cytoplasmic FAK-Dp71 and nucleus laminB1-Dp71 protein complex in HBE cells.This model shows, the localization of FAK-Dp71f/ Dp71d in the cytoplasm; the localization of lamin B1-Dp71f/Dp71d in the nucleus of HBE cells. Via increasing the binding proteins from transcription and translation level in these complexes, the HBE-Dp71f and HBEDp71d cells altered their biological characters.

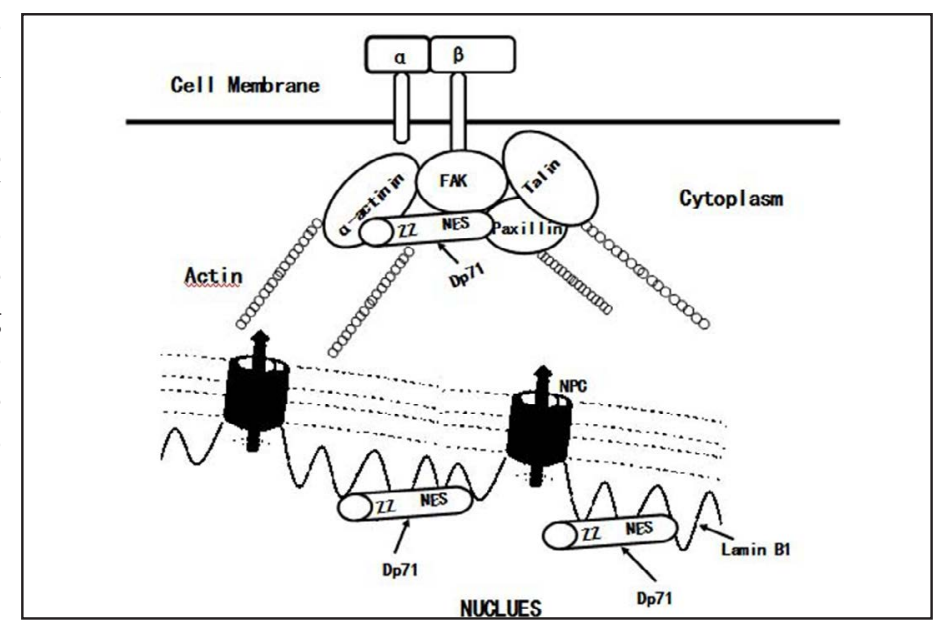

Dp71 in other cells identified both cytoplasmic and nucleus locations of these two Dp71 isoforms [30-32]. In order to explain that over-expressing of Dp71f or Dp71d results in similar phenotype alterations of HBE in our research. Dp71d and Dp71f plasmids with flag tagged Dp71 isoforms were constructed and transfected into HBE to track their subcellular locations. Our confocal results clearly show the enhanced nucleus and cytoplasmic Dp71 protein after the transfection of Dp71d-pcDNA3.1-3xFlag-C and Dp71f-pcDNA3.1-3xFlag-C plasmids. Considering the nucleus location of Dp71f in HEK293 and shuttling movements of Dp71d in PC12 cells, it is proposed that in HBE-Dp71f and HBE-Dp71d cells, by combining of cell-environmental factors and the Dp71 amino acid sequence, both Dp71d and Dp71f can enhance the nucleus and cytoplasmic DAPC proteins, which results in similar phenotype changes of these cells.

Bioinformatics analysis via Tess and Jaspar revealed that Dp71 is under the transcriptional control of quite a few transcription factors, such as HSF1 and Ap1. Our publication also proved that Dp71 is under positive regulation of HSF1 in vivo and in vitro [33]. Previous research proved that HSF1 and Ap1 increased their expression during ischemia reperfusion process [34-36]. Lies on the cross point of HSF1 and Ap1, Dp71 expression was expected to be increased under oxidative stress. Our preliminary work of Dp71 during cardiac and pulmonary ischemia-reperfusion process proved increased Dp71 expression in both organs (data not shown). Taking into account of the anti-apoptotic features of the HBE-Dp71d and HBE-Dp71f cells, it is concluded that enhanced Dp71 can play some anti-oxidant roles in the ischemia-reperfusion injury of different organs. However, more in vivo experiments need to be performed to verify this hypothesis. In summary, our work on HBE-Dp71d and HBEDp71f cells revealed some basic physiological roles such as proliferation, migration Dp71 played in human epithelium cells, which serves as an additional proof of the importance of this "old "cytoskeletal protein.

\section{Acknowledgements}

This study is supported by the National Natural Science Fund of China (Grant No. 30800550, 81300053), Hunan Natural Science Fund of China (Grant No.06C0410).

Plasmids expressing Dp71d and Dp71f isoforms were kind gifts offered by Dr Bulmaro Cisneros (Department of Genetics and Molecular biology, CINVESTAV-IPN, Mexico City).

\section{Disclosure Statement}

None. 


\section{Cellular Physiology Cell Physiol Biochem 2017;43:2022-2036

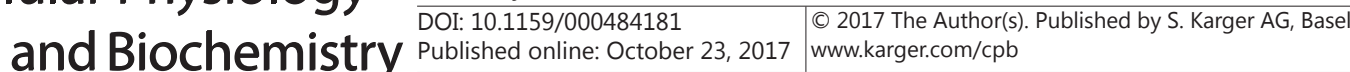

Tan et al.: Dp71 Enhanced HBE Cells

\section{References}

1 Blake DJ, Weir A, Newey SE, Davies KE: Function and genetics of dystrophin and dystrophin-related proteins in muscle. Physiol Rev 2002;82:291-329.

-2 Nowak KJ, Davies KE: Duchenne muscular dystrophy and dystrophin: pathogenesis and opportunities for treatment. EMBO Rep 2004;5:872-876.

-3 Howard PL, Dally GY, Ditta SD, Austin RC, Worton RG, Klamut HJ, Ray PN: Dystrophin isoforms DP71 and DP427 have distinct roles in myogenic cells. Muscle Nerve 1999;22:16-27.

-4 Austin RC, Howard PL, D’Souza VN, Klamut HJ, Ray PN: Cloning and characterization of alternatively spliced isoforms of Dp71. Hum Mol Genet 1995;4:1475-1483.

-5 Aragon J, Gonzalez-Reyes M, Romo-Yanez J, Vacca O, Aguilar-Gonzalez G, Rendon A, Vaillend C, Montanez C: Dystrophin Dp71 Isoforms Are Differentially Expressed in the Mouse Brain and Retina: Report of New Alternative Splicing and a Novel Nomenclature for Dp71 Isoforms. Mol Neurobiol 2017. doi: 10.1007/ s12035-017-0405-x.

6 Fabbrizio E, Nudel U, Hugon G, Robert A, Pons F, Mornet D: Characterization and localization of a $77 \mathrm{kDa}$ protein related to the dystrophin gene family. Biochem J 1994;299:359-365.

7 Tadayoni R, Rendon A, Soria-Jasso LE, Cisneros B: Dystrophin Dp71: the smallest but multifunctional product of the Duchenne muscular dystrophy gene. Mol Neurobiol 2012;45:43-60.

8 Sarig R, Mezger-Lallemand V, Gitelman I, Davis C, Fuchs O, Yaffe D, Nudel U: Targeted inactivation of Dp71, the major non-muscle product of the DMD gene: differential activity of the Dp71 promoter during development. Hum Mol Genet 1999;8:1-10.

- Bolanos-Jimenez F, Bordais A, Behra M, Strahle U, Sahel J, Rendon A: Dystrophin and Dp71, two products of the DMD gene, show a different pattern of expression during embryonic development in zebrafish. Mech Dev 2001;102:239-241.

10 Villarreal-Silva M, Centeno-Cruz F, Suarez-Sanchez R, Garrido E, Cisneros B: Knockdown of dystrophin Dp71 impairs PC12 cells cycle: localization in the spindle and cytokinesis structures implies a role for Dp71 in cell division. PLoS One 2011;6:e23504.

11 Acosta R, Montanez C, Fuentes-Mera L, Gonzalez E, Gomez P, Quintero-Mora L, Mornet D, Alvarez-Salas LM, Cisneros B: Dystrophin Dp71 is required for neurite outgrowth in PC12 cells. Exp Cell Res 2004;296:265275.

12 Rodriguez-Munoz R, Cardenas-Aguayo Mdel C, Aleman V, Osorio B, Chavez-Gonzalez O, Rendon A, MartinezRojas D, Meraz-Rios MA: Novel Nuclear Protein Complexes of Dystrophin 71 Isoforms in Rat Cultured Hippocampal GABAergic and Glutamatergic Neurons. PLoS One 2015;10:e0137328.

13 Fort PE, Sene A, Pannicke T, Roux MJ, Forster V, Mornet D, Nudel U, Yaffe D, Reichenbach A, Sahel JA, Rendon A: Kir4.1 and AQP4 associate with Dp71- and utrophin-DAPs complexes in specific and defined microdomains of Muller retinal glial cell membrane. Glia 2008;56:597-610.

14 Tan S, Tan J, Tan S, Zhao S, Cao X, Chen Z, Weng Q Zhang H, Wang K, Zhou J, Xiao X: Decreased Dp71 expression is associated with gastric adenocarcinoma prognosis. Oncotarget 2016;7:53702-53711.

15 Cerna J, Cerecedo D, Ortega A, Garcia-Sierra F, Centeno F, Garrido E, Mornet D, Cisneros B: Dystrophin Dp71f associates with the beta1-integrin adhesion complex to modulate PC12 cell adhesion. J Mol Biol 2006;362:954-965.

16 Rubi L, Gawali VS, Kubista H, Todt H, Hilber K, Koenig X: Proper Voltage-Dependent Ion Channel Function in Dysferlin-Deficient Cardiomyocytes. Cell Physiol Biochem 2015;36:1049-1058.

17 Aragon J, Romo-Yanez J, Martinez-Herrera A, Ceja V, Rendon A, Montanez C: Characterization of Dp71Delta(78-79), a novel dystrophin mutant that stimulates PC12 cell differentiation. J Neurochem 2011;119:697-707.

18 Beraud C, Dormoy V, Danilin S, Lindner V, Bethry A, Hochane M, Coquard C, Barthelmebs M, Jacqmin D, Lang H, Massfelder T: Targeting FAK scaffold functions inhibits human renal cell carcinoma growth. Int J Cancer 2015;137:1549-1559.

19 Wang D, Gao CQ, Chen RQ Jin CL, Li HC, Yan HC, Wang XQ: Focal adhesion kinase and paxillin promote migration and adhesion to fibronectin by swine skeletal muscle satellite cells. Oncotarget 2016;7:3084530854.

20 Cao W, Zheng W, Chen T: Ruthenium polypyridyl complex inhibits growth and metastasis of breast cancer cells by suppressing FAK signaling with enhancement of TRAIL-induced apoptosis. Sci Rep 2015;5:9157. 


\section{Cellular Physiology Cell Physiol Biochem 2017;43:2022-2036

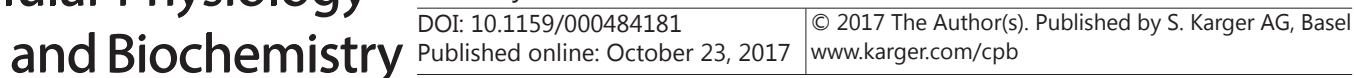 \\ Tan et al.: Dp71 Enhanced HBE Cells}

21 Huang D, Khoe M, Befekadu M, Chung S, Takata Y, Ilic D, Bryer-Ash M: Focal adhesion kinase mediates cell survival via NF-kappaB and ERK signaling pathways. Am J Physiol Cell Physiol 2007;292:C1339-1352.

- 22 Gharibi B, Ghuman MS, Hughes FJ: Akt- and Erk-mediated regulation of proliferation and differentiation during PDGFRbeta-induced MSC self-renewal. J Cell Mol Med 2012;16:2789-2801.

23 Cohen LA, Guan JL: Mechanisms of focal adhesion kinase regulation. Curr Cancer Drug Targets 2005;5:629643.

24 Chambard JC, Lefloch R, Pouyssegur J, Lenormand P: ERK implication in cell cycle regulation. Biochim Biophys Acta 2007;1773:1299-1310.

-25 Coqueret 0: New roles for p21 and p27 cell-cycle inhibitors: a function for each cell compartment? Trends Cell Biol 2003;13:65-70.

-26 Radeff-Huang J, Seasholtz TM, Chang JW, Smith JM, Walsh CT, Brown JH: Tumor necrosis factor-alphastimulated cell proliferation is mediated through sphingosine kinase-dependent Akt activation and cyclin D expression. J Biol Chem 2007;282:863-870.

27 De Castro SC, Malhas A, Leung KY, Gustavsson P, Vaux DJ, Copp AJ, Greene ND: Lamin b1 polymorphism influences morphology of the nuclear envelope, cell cycle progression, and risk of neural tube defects in mice. PLoS Genet 2012;8:e1003059.

28 Aleman V, Osorio B, Chavez O, Rendon A, Mornet D, Martinez D: Subcellular localization of Dp71 dystrophin isoforms in cultured hippocampal neurons and forebrain astrocytes. Histochem Cell Biol 2001;115:243254.

29 Gonzalez E, Montanez C, Ray PN, Howard PL, Garcia-Sierra F, Mornet D, Cisneros B: Alternative splicing regulates the nuclear or cytoplasmic localization of dystrophin Dp71. FEBS Lett 2000;482:209-214.

-30 Suarez-Sanchez R, Aguilar A, Wagstaff KM, Velez G, Azuara-Medina PM, Gomez P, Vasquez-Limeta A, Hernandez-Hernandez O, Lieu KG, Jans DA, Cisneros B: Nucleocytoplasmic shuttling of the Duchenne muscular dystrophy gene product dystrophin Dp71d is dependent on the importin alpha/beta and CRM1 nuclear transporters and microtubule motor dynein. Biochim Biophys Acta 2014;1843:985-1001.

-31 Nishida A, Yasuno S, Takeuchi A, Awano H, Lee T, Niba ET, Fujimoto T, Itoh K, Takeshima Y, Nishio H, Matsuo M: HEK293 cells express dystrophin Dp71 with nucleus-specific localization of Dp71ab. Histochem Cell Biol 2016;146:301-309.

-32 Rodriguez-Munoz R, Villarreal-Silva M, Gonzalez-Ramirez R, Garcia-Sierra F, Mondragon M, Mondragon R, Cerna J, Cisneros B: Neuronal differentiation modulates the dystrophin Dp71d binding to the nuclear matrix. Biochem Biophys Res Commun 2008;375:303-307.

33 Tan J, Tan S, Zheng H, Liu M, Chen G, Zhang H, Wang K, Tan S, Zhou J, Xiao XZ: HSF1 functions as a transcription regulator for Dp71 expression. Cell Stress Chaperones 2015;20:371-379.

-34 Zou Y, Zhu W, Sakamoto M, Qin Y, Akazawa H, Toko H, Mizukami M, Takeda N, Minamino T, Takano H, Nagai T, Nakai A, Komuro I: Heat shock transcription factor 1 protects cardiomyocytes from ischemia/reperfusion injury. Circulation 2003;108:3024-3030.

-35 Cho S, Park EM, Kim Y, Liu N, Gal J, Volpe BT, Joh TH: Early c-Fos induction after cerebral ischemia: a possible neuroprotective role. J Cereb Blood Flow Metab 2001;21:550-556.

-36 Zhang L, Jiang H, Gao X, Zou Y, Liu M, Liang Y, Yu Y, Zhu W, Chen H, Ge J: Heat shock transcription factor-1 inhibits H2O2-induced apoptosis via down-regulation of reactive oxygen species in cardiac myocytes. Mol Cell Biochem 2011;347:21-28. 